\title{
A HOMOLOGICAL RECIPE FOR PSEUDO-ANOSOVS
}

\author{
Dan Margalit and Steven Spallone
}

AbStract. We give a simple explicit construction of pseudo-Anosov mapping classes

using an improvement of the homological criterion of Casson-Bleiler.

\section{Introduction}

Starting from a homological criterion of Casson-Bleiler, we give a simple and explicit construction of pseudo-Anosov elements of the mapping class group. In the exposition, we relegate much of the standard background material about mapping class groups to other references; see, e.g., $[1,3]$.

Let $S$ be a surface of genus $g$. The mapping class group of $S$, denoted $\operatorname{Mod}(S)$, is the group of isotopy classes of homeomorphisms of $S$. The action of $\operatorname{Mod}(S)$ on $H_{1}(S, \mathbb{Z})$ is symplectic, and in fact $\operatorname{Mod}(S)$ surjects onto the integral symplectic group; we call this map $\Psi$. The kernel of $\Psi$ is called the Torelli group for $S$, and is denoted $\mathcal{I}(S)$. When $S$ has at most one boundary component, $H_{1}(S, \mathbb{Z}) \cong \mathbb{Z}^{2 g}$, and we have the following short exact sequence.

$$
1 \rightarrow \mathcal{I}(S) \rightarrow \operatorname{Mod}(S) \stackrel{\Psi}{\rightarrow} \operatorname{Sp}(2 g, \mathbb{Z}) \rightarrow 1
$$

Even given the Nielsen-Thurston classification, which says that every mapping class is periodic, reducible, or pseudo-Anosov, it is not at all obvious how to write down examples of pseudo-Anosov mapping classes. Thurston gave a simple explicit construction [8], in particular giving the first examples of pseudo-Anosov elements of $\mathcal{I}(S)$ (Nielsen conjectured that no such mapping classes exist).

Since that time, various constructions of pseudo-Anosov mapping classes have been given in the literature; we do not attempt to give a survey here. We do point out two advantages to our construction. First, our examples are very simple to write down as products of Dehn twists and "handle switches" (the latter can in turn be written as simple products of Dehn twists). Second, since we are using a homological criterion, our construction is robust in the sense that if $f$ is one of our examples and $f^{\prime} \in \mathcal{I}(S)$, then $f f^{\prime}$ is pseudo-Anosov.

Bounding triple maps and handle switches. Our construction involves two types of elementary mapping classes: bounding triple maps and handle switches. Let $S$ be a surface of genus $g$ with at most one boundary component, and let $\left\{x_{i}, y_{i}\right\}$ be a standard symplectic basis for $H_{1}(S, \mathbb{Z})$, as in Figure 1.

Received by the editors September 22, 2006.

2000 Mathematics Subject Classification. Primary: 20F36; Secondary: 57M07.

Key words and phrases. pseudo-Anosov, mapping class groups.

The first author gratefully acknowledges support from the National Science Foundation. 


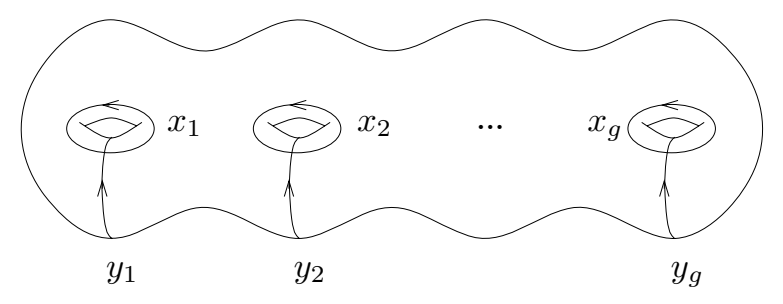

Figure 1. A standard symplectic basis for $H_{1}(S, \mathbb{Z})$.

For each $1 \leq i \leq g$, choose a mapping class $\mathcal{B}_{i}$ as follows.

$$
\mathcal{B}_{i}= \begin{cases}T_{x_{i}+x_{i+1}}^{-1} T_{x_{i}} T_{x_{i+1}} & i<g, i \text { odd } \\ T_{y_{i}+y_{i+1}} T_{y_{i}}^{-1} T_{y_{i+1}}^{-1} & i<g, i \text { even } \\ T_{x_{i}}^{-1} & i=g, i \text { odd } \\ T_{y_{i}} & i=g, i \text { even }\end{cases}
$$

As usual, $T_{a}$ denotes the (left) Dehn twist about a curve $a$ (we are confusing homology classes with curves in the definition; any curve in the given homology class will suffice). Each $\mathcal{B}_{i}$ with $i<g$ is what we call a bounding triple map, for each can be realized as a product of Dehn twists about three mutually disjoint curves, no pair of which separates $S$, but the union of which does.

By a handle switch $\mathcal{H}_{i}$, we will mean any mapping class which achieves the following action on $H_{1}(S, \mathbb{Z})$. As a convention, all vectors which do not appear in such a formula are fixed.

$$
\begin{aligned}
& \left(x_{i}, y_{i}, x_{i+1}, y_{i+1}\right) \mapsto \begin{cases}\left(-y_{i+1}, x_{i+1},-y_{i}, x_{i}\right) & i<g, i \text { odd } \\
\left(-y_{i+1}, x_{i+1},-y_{i}, x_{i}\right) & i<g, i \text { even }\end{cases} \\
& \left(x_{i}, y_{i}\right) \mapsto \begin{cases}\left(-y_{i}, x_{i}\right) & i=g, i \text { odd } \\
\left(y_{i},-x_{i}\right) & i=g, i \text { even }\end{cases}
\end{aligned}
$$

We give two ways of realizing a handle switch in Section 3.3.

Symmetrization of polynomials. Our construction requires one more ingredient. We define a function Sym : $\mathbb{Z}[x] \rightarrow \mathbb{Z}[x]$ via the following formula.

$$
\operatorname{Sym}(q)(x)=x^{\operatorname{deg}(q)} \cdot q\left(x+\frac{1}{x}\right)
$$

The use of this function was suggested by Zrinka Despotovic.

Statement of the construction. We now are ready to describe a typical pseudoAnosov mapping class yielded by our construction. Let $S, \mathcal{B}_{i}$, and $\mathcal{H}_{i}$ be as above.

Let $p$ be any prime number, and let $a_{0}, \ldots, a_{g-1}$ be integers with $a_{0}$ not divisible by $p$ and $\left|a_{g-1} p\right|>2 g$ (recall $g$ is the genus of $S$ ). Consider the polynomial

$$
q(x)=x^{g}+a_{g-1} p x^{g-1}+\cdots+a_{0} p
$$

and define integers $c_{i}$ via the following formula.

$$
\operatorname{Sym}(q)(x)=x^{2 g}+c_{1} x^{2 g-1}+\cdots+c_{2 g-1} x+1
$$


Finally, let $t_{i}=(-1)^{i} c_{i}$, and consider the following mapping class.

$$
f=\mathcal{B}_{1}^{t_{1}} \mathcal{H}_{1} \cdots \mathcal{B}_{g-1}^{t_{g-1}} \mathcal{H}_{g-1} \mathcal{B}_{g}^{t_{g}} \mathcal{H}_{g}
$$

Theorem. Each element of the coset $f \mathcal{I}(S)$ is pseudo-Anosov.

Questions. Building on a construction of Thurston, Penner gave the following easyto-state (and powerful) construction of pseudo-Anosovs [5]. Let $A=\left\{a_{i}\right\}$ and $B=$ $\left\{b_{i}\right\}$ be two multicurves which fill a surface $S$, and let $f$ be any mapping class obtained as a product of positive powers of the $T_{a_{i}}$ and negative powers of the $T_{b_{i}}$ (each $a_{i}$ and $b_{i}$ must appear at least once). Penner's theorem is that $f$ is pseudo-Anosov. Penner has conjectured that every pseudo-Anosov mapping class has a power which is given by this construction. Thus, it is natural to ask whether or not this is true for our examples. Even if we write one such $f$ in "Penner form," it is not clear how to write the other elements of $f \mathcal{I}(S)$ in this way.

For any of our examples $f$, we would like to know if there is an element of $f \mathcal{I}(S)$ whose stable and unstable foliations are orientable. In that case, the dilatation is exactly the spectral radius of the matrix $\Psi(f)$. It may be possible to gain insight into the spectrum of dilatations of $\operatorname{Mod}(S)$ in this way. For instance, Farb-LeiningerMargalit showed that the minimal dilatation pseudo-Anosov in $\operatorname{Mod}(S)$ does not lie in any of the level $m$ congruence subgroups for $m \geq 3$ [2]; our construction yields examples which do not lie in any such subgroup. Chris Leininger has pointed out the following two facts: only finitely many $\operatorname{Mod}(S)$ conjugacy classes in a $\mathcal{I}(S)$ coset can have orientable foliations (since in this case the dilatations are all the same, and it is known that there are only finitely many conjugacy classes of pseudo-Anosov elements with dilatation less than any given constant); also, since there are "CassonBleiler polynomials" with no real roots, we have examples of cosets where there are no representatives with orientable foliations.

\section{The homological criterion}

In "Automorphisms of surfaces after Nielsen and Thurston," Casson-Bleiler proved the following fact [1, Lemma 5.1].

Proposition 1. Let $f \in \operatorname{Mod}(S)$, let $q_{f}(x)$ be the characteristic polynomial for $\Psi(f)$. If $q_{f}(x)$ is irreducible over $\mathbb{Z}$, has no roots of unity as zeros, and is not a polynomial in $x^{k}$ for $k>1$, then $f$ is pseudo-Anosov.

We remark that the only example to which Casson-Bleiler apply this homological criterion is a product of Dehn twists which (as they point out) is a case of the Penner construction mentioned earlier. Below, we give a refinement of Proposition 1 which lends itself better to an explicit construction of pseudo-Anosov mapping classes; indeed, the examples given in the introduction do not in general satisfy the hypotheses of Proposition 1.

We first need to introduce some terminology pertaining to polynomials. A (integer) symplectic polynomial is any polynomial which is the characteristic polynomial of some element of $\operatorname{Sp}(2 n, \mathbb{Z})$. It is a fact that an even degree integer polynomial

$$
q(x)=a_{2 n} x^{2 n}+\cdots+a_{1} x+a_{0}
$$


is a symplectic polynomial if and only if it is monic $\left(a_{2 n}=1\right)$ and palindromic $\left(a_{i}=a_{2 n-i}\right.$ for $\left.0 \leq i \leq n\right)$. We say that a symplectic polynomial is symplectically irreducible if it is not the product of two nontrivial symplectic polynomials.

Upon examination of the proof of Proposition 1 (see [1]), we see that we can replace the word "irreducible" with "symplectically irreducible"; this fact was pointed out to us by Mladen Bestvina. Also, as noticed by Silver-Williams, we can replace the assumption that $p_{f}(x)$ has no roots of unity as zeros with the assumption that $q_{f}(x)$ is not cyclotomic [6].

Proposition 2 (Homological criterion). Let $S$ be a closed surface of genus at least 2, let $f \in \operatorname{Mod}(S)$, and let $q_{f}(x)$ be the characteristic polynomial for $\Psi(f)$. If $q_{f}(x)$ is symplectically irreducible, is not a cyclotomic polynomial, and is not a polynomial in $x^{k}$ for $k>1$, then $f$ is pseudo-Anosov.

The hypotheses of Proposition 2 can be thought of as hypotheses on the mapping class $f$, the matrix $\Psi(f)$, or the polynomial $q_{f}(x)$. We will say that a mapping class, matrix, or polynomial satisfies the homological criterion if it (or the associated polynomial) satisfies the hypotheses of the proposition.

Briefly, Proposition 2 is explained as follows. If a mapping class $f$ is periodic, then $\Psi(f)$ is periodic, and so $q_{f}(x)$ has only roots of unity as zeros. If $f$ is reducible and $f^{k}$ fixes a nonseparating curve, then $q_{f}$ has a root of unity as a zero. If $f$ is reducible and $f$ fixes a collection of separating curves, then either $f$ preserves two distinct subsurfaces of positive genus (in which case $q_{f}$ is symplectically reducible) or $f$ transitively permutes a collection of subsurfaces which span $H_{1}(S, \mathbb{Z}$ ) (in which case the matrix has several identity blocks and so $q_{f}$ is a polynomial in $x^{k}$ for some $k>1$ ). One also needs the remark that a symplectically irreducible polynomial which has a root of unity as a zero is necessarily cyclotomic. Thus, if $q_{f}$ satisfies the homological criterion, then $f$ is neither periodic nor reducible; by the NielsenThurston classification, $f$ is pseudo-Anosov.

\section{The construction}

We will use the homological criterion to give a construction of pseudo-Anosov mapping classes in the following way:

(1) For every positive integer $g$, we give an explicit construction of infinitely many symplectic polynomials of degree $2 g$, each of which satisfies the homological criterion.

(2) Given any symplectic polynomial $q(x)$ of degree $2 g$, we give an element $A_{q}$ of $\mathrm{Sp}(2 g, \mathbb{Z})$ with characteristic polynomial $q(x)$.

(3) For each such element $A_{q}$ of $\operatorname{Sp}(2 g, \mathbb{Z})$, we give an infinite collection of mapping classes whose action on $H_{1}(S, \mathbb{Z})$ is given by $A_{q}$.

Each of the three steps is detailed below. In Section 4, we give a complete description of the situation for genus 2 surfaces.

3.1. Polynomials. The first step is to find polynomials which satisfy the homological criterion. Recall the function Sym : $\mathbb{Z}[x] \rightarrow \mathbb{Z}[x]$ defined in the introduction. We see that if $q(x)$ is monic, then $\operatorname{Sym}(q)(x)$ is monic, of even degree, and is palindromic, i.e., $\operatorname{Sym}(q)(x)$ is symplectic. 
It is an elementary fact that the function Sym : $\mathbb{Z}[x] \rightarrow \mathbb{Z}[x]$ is multiplicative, and injective. Further, the monic elements surject onto the symplectic polynomials. We thus have the following.

Lemma 3. If $q(x) \in \mathbb{Z}[x]$ is monic, then $\operatorname{Sym}(q)$ is symplectically irreducible if and only if $q(x)$ is irreducible over $\mathbb{Z}$.

From the definition of $\operatorname{Sym}$, we see that $\zeta$ is a root of $\operatorname{Sym}(q)$ if and only if $\zeta+\frac{1}{\zeta}$ is a root of $q$. The following is a consequence.

Lemma 4. If $\operatorname{Sym}(q)(x)$ is a cyclotomic polynomial, then each root of $q(x)$ has absolute value bounded above by 2 .

By a straightforward computation, we see that the coefficient of $x^{g-1}$ in a degree $g$ polynomial $q(x)$ is equal to the coefficients of $x$ and $x^{2 g-1}$ in $\operatorname{Sym}(q)(x)$. We thus have the following.

Lemma 5. If $q(x) \in \mathbb{Z}[x]$ is a monic degree $g$ polynomial, and the coefficient of $x^{g-1}$ is nonzero, then $\operatorname{Sym}(q)(x)$ is not a polynomial in $x^{k}$ for any $k>1$.

Proposition 6. Let $q(x) \in \mathbb{Z}[x]$ be an irreducible polynomial of the following form.

$$
q(x)=x^{g}+a_{g-1} x^{g-1}+\cdots+a_{1} x+a_{0}
$$

If $\left|a_{g-1}\right|>2 g$, then $\operatorname{Sym}(q)(x)$ satisfies the homological criterion.

Proof. By Lemma 3, $\operatorname{Sym}(q)(x)$ is symplectically irreducible. The condition on $a_{g-1}$ implies that some root of $q$ has absolute value greater than 2 (indeed, $-a_{g-1}$ is the sum of the roots of $q$ ); so by Lemma 4 we see that $\operatorname{Sym}(q)(x)$ is not a cyclotomic polynomial. Finally, by Lemma $5, \operatorname{Sym}(q)(x)$ is not a polynomial in $x^{k}$ for any $k>1$.

We can now use the Eisenstein criterion to construct explicit families of polynomials which satisfy the homological criterion.

Proposition 7. Let $p$ be a prime number, and let $a_{g-1}, \ldots, a_{0}$ be integers with $\left|a_{g-1} p\right|>2 g$ and $a_{0}$ not divisible by $p$. If we set

$$
q(x)=x^{g}+a_{g-1} p x^{g-1}+\cdots+a_{0} p
$$

then $\operatorname{Sym}(q)$ satisfies the homological criterion.

3.2. Matrices. We give the definition of the symplectic group and give two types of basic elements: symplectic elementary matrices and symplectic elementary permutation matrices. We then use the theory of rational canonical forms to give a symplectic matrix with any given symplectic polynomial as its characteristic polynomial, and to factor the matrix into a product of matrices of the above two types. 
Symplectic matrices. To establish notation, we set

$$
J=\left(\begin{array}{ccccc}
0 & 0 & \cdots & 0 & 1 \\
0 & 0 & \cdots & -1 & 0 \\
\vdots & \vdots & \ddots & \vdots & \vdots \\
0 & 1 & \cdots & 0 & 0 \\
-1 & 0 & \cdots & 0 & 0
\end{array}\right)
$$

and say that a matrix $A$ is symplectic when the following holds.

$$
A^{T} J A=J
$$

Let $\left\{e_{i}\right\}$ be the standard basis vectors for $\mathbb{Z}^{2 g}$. Using the inner product defined by $J$, we see that $\left\langle e_{i}, e_{2 g-i+1}\right\rangle=(-1)^{i+1}$ and all other inner products are zero; thus the $\left\{e_{i}\right\}$, in the order $e_{1}, e_{2 g}, e_{2 g-1}, e_{2}, e_{3}, e_{2 g-2}, \ldots$ give a symplectic basis for $\mathbb{Z}^{2 g}$. We denote these vectors by $x_{1}, y_{1}, x_{2}, y_{2}, \ldots, x_{g}, y_{g}$, respectively. Let $\sigma$ be the permutation of $\{1, \ldots, 2 g\}$ which is the product of the (commuting) transpositions $i \leftrightarrow 2 g-i+1$ for $1 \leq i \leq g$; note that $\left\{e_{i}, e_{\sigma(i)}\right\}=\left\{x_{i}, y_{i}\right\}$.

There are two types of elementary symplectic matrices. If $i=\sigma(j)$, then the symplectic elementary matrix $\mathrm{SE}_{i, j}$ is given by the row operation

$$
e_{i} \mapsto e_{i}+e_{j}
$$

(that is, add the $j^{\text {th }}$ row to the $i^{\text {th }}$ row; again, in general, when we write a row operation, the unmentioned rows are fixed). If $i \neq \sigma(j)$, then the elementary symplectic matrix $\mathrm{SE}_{i, j}$ is given by the following row operations.

$$
\begin{aligned}
e_{i} & \mapsto e_{i}+e_{j} \\
e_{\sigma(j)} & \mapsto e_{\sigma(j)}+(-1)^{i+j+1} e_{\sigma(i)}
\end{aligned}
$$

It is a fact that the $\mathrm{SE}_{i, j}$ generate the symplectic group $\operatorname{Sp}(2 g, \mathbb{Z})$.

Let $N_{i, j}$ denote the matrix given by the following row operation.

$$
\left(e_{i}, e_{j}\right) \mapsto\left(-e_{j}, e_{i}\right)
$$

We then have the symplectic elementary permutation matrices $W_{i, j}$. If $i=\sigma(j)$, set

$$
W_{i, j}=N_{i, j}
$$

and otherwise

$$
W_{i, j}=N_{i, j} N_{\sigma(j), \sigma(i)}
$$

Matrices with a given characteristic polynomial. Let $q(x)$ be the element $x^{2 g}+$ $c_{1} x^{2 g-1}+\cdots+c_{2 g}$ of $\mathbb{Z}[x]$, and consider the following matrix.

$$
A_{q}=\left(\begin{array}{rrrrr}
-c_{1} & -c_{2} & \cdots & -c_{2 g-1} & -c_{2 g} \\
1 & 0 & \cdots & 0 & 0 \\
0 & 1 & \cdots & 0 & 0 \\
\vdots & \vdots & \ddots & \vdots & \vdots \\
0 & 0 & \cdots & 1 & 0
\end{array}\right)
$$

Lemma 8. The matrix $A_{q}$ has characteristic polynomial $q(x)$. 
If the determinant of $A_{q}$ (namely $c_{2 g}$ ) is equal to 1 , one can obtain $A_{q}$ as a product of elementary matrices and permutation matrices in a simple way. Let $t_{i}=(-1)^{i} c_{i}$. Write $X_{i}$ for the elementary matrix corresponding to the row operation $e_{i} \mapsto e_{i}+$ $t_{i} e_{i+1}, N_{i}$ for $N_{i, i+1}$, and $Y_{i}$ for $X_{i} N_{i}$. We have the following theorem $[7, \S 7.4, \S 7.8]$.

Theorem 9. Using the notation above, we have

$$
A_{q}=Y_{1} Y_{2} \cdots Y_{2 g-1}
$$

Moreover, if the $Y_{i}$ are multiplied in a different order, the result is conjugate to $A_{q}$, and thus has the same characteristic polynomial (namely, $q(x)$ ).

Consider the following expression.

$$
B_{q}=\left(Y_{1} Y_{2 g-1}\right)\left(Y_{2} Y_{2 g-2}\right) \cdots\left(Y_{g-1} Y_{g+1}\right) Y_{g}
$$

Note that

$$
Y_{i} Y_{2 g-i}=X_{i} N_{i} X_{2 g-i} N_{2 g-i}=\left(X_{i} X_{2 g-i}\right)\left(N_{i} N_{2 g-i}\right)
$$

since $N_{i}$ and $X_{2 g-i}$ commute. If $q(x)$ is symplectic, then the matrices $X_{i} X_{2 g-i}$, $N_{i} N_{2 g-i}$, and $Y_{g}=X_{g} N_{g}$ are symplectic. Indeed, for $i<g$, we have

$$
\begin{aligned}
X_{i} X_{2 g-i} & =\mathrm{SE}_{i, i+1}^{t_{i}} \\
N_{i} N_{2 g-i} & =W_{i, i+1}
\end{aligned}
$$

and also

$$
\begin{aligned}
X_{g} & =\mathrm{SE}_{g, g+1}^{t_{g}} \\
N_{g} & =W_{g, g+1}
\end{aligned}
$$

Thus we have expressed $B_{q}$, which is conjugate to $A_{q}$ (Theorem 9 ), explicitly as a product of symplectic elementary matrices and symplectic elementary permutation matrices.

$$
B_{q}=\mathrm{SE}_{1,2}^{t_{1}} W_{1,2} \mathrm{SE}_{2,3}^{t_{2}} W_{2,3} \cdots \mathrm{SE}_{g-1, g}^{t_{g-1}} W_{g-1, g} \mathrm{SE}_{g, g+1}^{t_{g}} W_{g, g+1}
$$

For our purposes, this is convenient, because it is not difficult to find simple, explicit mapping classes whose actions on homology are given by $\mathrm{SE}_{i, i+1}$ and $W_{i, i+1}$.

Remark. One could also apply the "Steinberg section" [4] to the symplectic group to immediately obtain the same expression for $B_{q}$.

3.3. Mapping classes. To this point, we have given explicit examples of symplectic polynomials which satisfy the homological criterion, and we have produced explicit matrices with the given polynomials as characteristic polynomials. Moreover, we have given these matrices as products of the symplectic elementary matrices $\mathrm{SE}_{i, j}$ and symplectic elementary permutation matrices $W_{i, j}$. Thus, to complete our construction of pseudo-Anosov mapping classes, it suffices to exhibit mapping classes whose actions on $H_{1}(S, \mathbb{Z})$ are given by the $\mathrm{SE}_{i, j}$ and $W_{i, j}$. Again, we use the standard basis for $H_{1}(S, \mathbb{Z})$ shown in Figure 1.

If $x$ is an element of $H_{1}(S, \mathbb{Z})$, then we write $T_{x}$ for the Dehn twist (to the left) about any simple closed curve representing $x$. As a result of this convention, we will not be specifying particular elements of $\operatorname{Mod}(S)$ below, but rather cosets of $\mathcal{I}(S)$, from which particular elements are easily extracted. 
For $1 \leq i, j \leq 2 g$, set $t=(-1)^{j+1}$, and define

$$
\mathcal{B}_{i, j}= \begin{cases}T_{e_{i}}^{t} & i=\sigma(j) \\ \left(T_{e_{i}+e_{\sigma(j)}} T_{e_{i}}^{-1} T_{e_{\sigma(j)}}^{-1}\right)^{t} & i \neq \sigma(j)\end{cases}
$$

(Recall that the $e_{i}$ are the standard basis elements for $\mathbb{Z}^{2 g} \cong H_{1}(S, \mathbb{Z})$ and are identified with the $x_{i}$ and $y_{i}$ as in Section 3.2.) In the case $j=i+1$, we get the mapping classes $\mathcal{B}_{i}$ given in the introduction.

Lemma 10. For any $i$ and $j, \Psi\left(\mathcal{B}_{i, j}\right)=S E_{i, j}$.

By a change of coordinates, it suffices to check Lemma 10 for a genus 2 surface and the 4 possibilities for $i$ and $j$ even and odd.

We remark that Lemma 10, combined with the fact that the $\mathrm{SE}_{i, j}$ generate the group $\operatorname{Sp}(2 g, \mathbb{Z})$, constitutes a proof of the classical theorem that $\Psi: \operatorname{Mod}(S) \rightarrow$ $\operatorname{Sp}(2 g, \mathbb{Z})$ is surjective.

For $i=\sigma(j), W_{i, j}$ corresponds to the following signed row permutation.

$$
\left(e_{i}, e_{\sigma(i)}\right) \mapsto\left(-e_{\sigma(i)}, e_{i}\right)
$$

Such a mapping class can be visualized as a quarter-turn of a genus one subsurface spanned homologically by $e_{i}$ and $e_{\sigma(i)}$; see Figure 2. As a product of Dehn twists, this is the following mapping class.

$$
\mathcal{Q}_{i}=\left(T_{e_{i}} T_{e_{\sigma(i)}} T_{e_{i}}\right)^{(-1)^{i+1}}
$$

When $i=g$, we denote this map by $\mathcal{H}_{g}$ (to be consistent with the introduction).
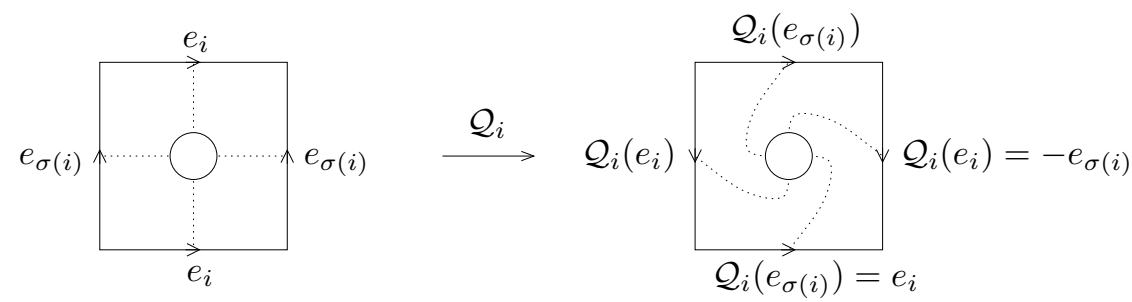

FiguRE 2. Realizing $W_{i, \sigma(i)}$ as $\mathcal{Q}_{i}$, a quarter turn of a handle. Identifying sides with the same labels, each picture represents a torus with one boundary component (the circle). We imagine the rest of the surface glued along the circle. The mapping class is determined by the data in the figure; the circle and the complement of this handle are fixed pointwise. The dotted arcs are shown for reference.

If $i \neq \sigma(j)$, the matrix $W_{i, j}$ is given by the following signed row permutation.

$$
\left(e_{i}, e_{\sigma(i)}, e_{j}, e_{\sigma(j)}\right) \mapsto\left(-e_{j}, e_{\sigma(j)}, e_{i},-e_{\sigma(i)}\right)
$$

In order to construct a mapping class whose action on homology is a permutation matrix, one can choose standard representatives for the $e_{k}$, cut $S$ along each $e_{k}$ in order to obtain a surface $S^{\prime}$ with $g$ "square" boundary components, take any homeomorphism of $S^{\prime}$ where the action on the squares is given by the action on homology, and then "glue up" to get a homeomorphism of $S$. 
If one prefers, it is also possible to find an explicit product of Dehn twists which does the same job. For simplicity, we consider the case where $i$ and $j$ have different parity and neither is equal to $g$ (this suffices for the construction). Let $\mathcal{H}_{i}$ be the mapping class

$$
\left(\left(T_{e_{\sigma(i)}} T_{e_{i}} T_{e_{\sigma(i)}+e_{i+1}} T_{e_{\sigma(i+1)}} T_{e_{i+1}}\right)^{3}\left(T_{e_{\sigma(i)}} T_{e_{i}}\right)^{3}\right)^{t}
$$

where $t=(-1)^{i+1}$ (the first factor switches the two handles and the second factor turns one of the handles by $\left.180^{\circ}\right)$.

Lemma 11. For $1 \leq i \leq g, \Psi\left(\mathcal{H}_{i}\right)=W_{i, i+1}$.

Again, this lemma can be checked by reducing the case of $4 \times 4$ matrices (genus 2 surfaces).

By Lemmas 10 and 11, one can verify that the construction given in the introduction is correct. The most general statement we will make is as follows.

Theorem. Let $S$ be a surface of genus $g$ with at most one boundary component. Let $q(x)=x^{g}+a_{g-1} x^{g-1}+\cdots+a_{0}$ be a monic degree $g$ irreducible integral polynomial with $\left|a_{g-1}\right|>2 g$. Define integers $c_{i}$ via

$$
\operatorname{Sym}(q)(x)=x^{2 g}+c_{1} x^{2 g-1}+\cdots+c_{2 g-1} x+1
$$

and let $t_{i}=(-1)^{i} c_{i}$. Let $f$ be a product of the elements of $\left\{\left(\mathcal{B}_{i}^{t_{i}} \mathcal{H}_{i}\right)\right\}_{i=1}^{g}$, where $\mathcal{H}_{i}$ and $\mathcal{B}_{i}$ are as in the introduction, and the product is taken in any order. In this case, each element of the coset $f \mathcal{I}(S)$ is pseudo-Anosov.

Remark. Kanenobu has found, for each symplectic polynomial, a fibered link whose associated monodromy has that polynomial as the characteristic polynomial of the action of the monodromy on the fiber surface [4]. However, we do not know how to explicitly understand these monodromies topologically on the surface.

\section{Genus 2}

Let $S$ be the closed orientable surface of genus 2. We proceed in the same general outline as in Section 3. That is, we first say exactly when a degree 4 symplectic polynomial satisfies the homological criterion, then give an explicit product of matrices for each symplectic polynomial, and finally give an explicit and simple product of Dehn twists corresponding to the product of matrices.

Proposition 12. A degree 4 symplectic polynomial

$$
q(x)=x^{4}+a x^{3}+b x^{2}+a x+1
$$

satisfies the homological criterion if and only if $a \neq 0,(a, b)$ is not $(1,1)$ or $(-1,1)$, and $a^{2}-4 b+8$ is not a perfect square.

Proof. It is straightforward to check that $q(x)=\operatorname{Sym}(h(x))$, where

$$
h(x)=x^{2}+a x+(b-2)
$$

By Lemma 3, $q(x)$ is symplectically reducible if and only if $h(x)$ is reducible over $\mathbb{Z}$. By the quadratic formula, this is exactly when $-a \pm \sqrt{a^{2}-4 b+8}$ is an even integer. Note that this is always the case when the discriminant is a perfect square. By checking the list of degree 4 cyclotomic polynomials, $q(x)$ is cyclotomic exactly 
when $(\mathrm{a}, \mathrm{b})$ is $(1,1),(0,0)$, or $(-1,1)$. Finally, $q(x)$ is a polynomial in $x^{4}$ or $x^{2}$ exactly when $a=0$.

As in Section 3.2, the matrix

$$
A_{q}=\left(\begin{array}{rrrr}
-a & -b & -a & -1 \\
1 & 0 & 0 & 0 \\
0 & 1 & 0 & 0 \\
0 & 0 & 1 & 0
\end{array}\right)
$$

has characteristic polynomial $q(x)=x^{4}+a x^{3}+b x^{2}+a x+1$ (this can be checked by direct computation).

As per Section 3.2, we consider the product

$$
\left(X_{1} X_{3}\right)\left(N_{1} N_{3}\right)\left(X_{2}\right)\left(N_{2}\right)=\mathrm{SE}_{1,2}^{-a} W_{1,3} \mathrm{SE}_{2,3}^{b} W_{2,3}
$$

which is equal to the following.

$$
\left(\begin{array}{rrrr}
1 & -a & 0 & 0 \\
0 & 1 & 0 & 0 \\
0 & 0 & 1 & -a \\
0 & 0 & 0 & 1
\end{array}\right)\left(\begin{array}{rrrr}
0 & -1 & 0 & 0 \\
1 & 0 & 0 & 0 \\
0 & 0 & 0 & -1 \\
0 & 0 & 1 & 0
\end{array}\right)\left(\begin{array}{llll}
1 & 0 & 0 & 0 \\
0 & 1 & b & 0 \\
0 & 0 & 1 & 0 \\
0 & 0 & 0 & 1
\end{array}\right)\left(\begin{array}{rrrr}
1 & 0 & 0 & 0 \\
0 & 0 & -1 & 0 \\
0 & 1 & 0 & 0 \\
0 & 0 & 0 & 1
\end{array}\right)
$$

This last product is conjugate to $A_{q}$ and hence has characteristic polynomial $q(x)$. We are free to change the signs of the middle two columns in the second and fourth matrices since this operation has order 2 and commutes with the third matrix.

$$
\left(\begin{array}{rrrr}
1 & -a & 0 & 0 \\
0 & 1 & 0 & 0 \\
0 & 0 & 1 & -a \\
0 & 0 & 0 & 1
\end{array}\right)\left(\begin{array}{rrrr}
0 & 1 & 0 & 0 \\
1 & 0 & 0 & 0 \\
0 & 0 & 0 & -1 \\
0 & 0 & -1 & 0
\end{array}\right)\left(\begin{array}{llll}
1 & 0 & 0 & 0 \\
0 & 1 & b & 0 \\
0 & 0 & 1 & 0 \\
0 & 0 & 0 & 1
\end{array}\right)\left(\begin{array}{rrrr}
1 & 0 & 0 & 0 \\
0 & 0 & 1 & 0 \\
0 & -1 & 0 & 0 \\
0 & 0 & 0 & 1
\end{array}\right)
$$

These four matrices, from left to right, are the images of the mapping classes $\left(T_{x_{1}+x_{2}} T_{x_{1}}^{-1} T_{x_{2}}^{-1}\right)^{a},\left(T_{y_{2}} T_{x_{2}} T_{y_{1}+y_{2}} T_{x_{1}} T_{y_{1}}\right)^{3}, T_{y_{2}}^{b}$, and $T_{x_{2}} T_{y_{2}} T_{x_{2}}$, respectively.

Proposition 13. If $a$ and $b$ are integers with the properties that $a \neq 0,(a, b)$ is not $(1,1)$ or $(1,-1)$ and $a^{2}-4 b+8$ is not a perfect square, and $f$ is any element of $\mathcal{I}(S)$, then the mapping class

$$
\left(T_{x_{1}+x_{2}} T_{x_{1}}^{-1} T_{x_{2}}^{-1}\right)^{a}\left(T_{y_{2}} T_{x_{2}} T_{y_{1}+y_{2}} T_{x_{1}} T_{y_{1}}\right)^{3}\left(T_{y_{2}}\right)^{b}\left(T_{x_{2}} T_{y_{2}} T_{x_{2}}\right) f
$$

is pseudo-Anosov.

\section{Acknowledgements}

The authors are grateful to Ian Agol, Mladen Bestvina, Joan Birman, Zrinka Despotovic, Sam Evens, Benson Farb, Misha Kapovich, Chris Leininger, Lee Mosher, Dan Silver, Susan Williams, and the referee for their comments and help. 


\section{References}

[1] A. J. Casson and S. A. Bleiler, Automorphisms of surfaces after Nielsen and Thurston, Vol. 9 of London Mathematical Society Student Texts, Cambridge University Press, Cambridge (1988), ISBN 0-521-34203-1.

[2] B. Farb, C. J. Leininger, and D. Margalit, The lower central series and pseudo-Anosov dilatations. arXiv:math.GT/0603675.

[3] B. Farb and D. Margalit, A primer on mapping class groups. In preparation.

[4] T. Kanenobu, Module d'Alexander des nœuds fibrés et polynôme de Hosokawa des lacements fibrés, Math. Sem. Notes Kobe Univ. 9 (1981), no. 1, 75-84.

[5] R. C. Penner, A construction of pseudo-Anosov homeomorphisms, Trans. Amer. Math. Soc. 310 (1988), no. 1, 179-197.

[6] D. S. Silver and S. G. Williams, Lehmer's question, knots and surface dynamics. ArXiv:math.GT/0509068. To appear in Mathematical Proceedings of the Cambridge Philosophical Society.

[7] R. Steinberg, Regular elements of semisimple algebraic groups, Inst. Hautes Études Sci. Publ. Math. (1965), no. 25, 49-80.

[8] W. P. Thurston, On the geometry and dynamics of diffeomorphisms of surfaces, Bull. Amer. Math. Soc. (N.S.) 19 (1988), no. 2, 417-431.

Department of Mathematics, University of Utah, 155 S 1440 East, Salt Lake City, Ut 84112-0090

E-mail address: margalit@math.utah.edu

Department of Mathematics, Purdue University, 150 N. University Street, West LAFAYETTE, , IN 47907-2067

E-mail address: sspallon@math.purdue.edu 